Génét. Sél. Evol., 1988, 20 (1), 105-110

\title{
Note
}

\section{Effets du gène "crête en pois " et d'autres gènes à effet visible sur le poids des coqs adultes}

\author{
P. MÉRAT \\ Institut National de la Recherche Agronomique, \\ Centre de Recherches de Jouy-en-Josas, \\ Laboratoire de Génétique Factorielle, 78350 Jouy-en-Josas, France
}

\begin{abstract}
Résumé
Dans un troupeau expérimental du type "pondeuse à œufs teintés » mi-lourd, de 1961 à 1984, des génotypes relatifs à des locus de coloration ou à effet morphologique ont été comparés sur des couples de frères ou demi-frères. Sur 42 couples de coqs $\mathrm{Pp}^{+}$(crête en pois) et $\mathrm{p}^{+} \mathrm{p}^{+}$(crête simple), le poids moyen des premiers à 40 semaines dépasse celui des seconds d'environ $250 \mathrm{~g}$, soit $7,8 \%$ du poids moyen du génotype homozygote récessif $(P<0,001)$. Cette différence provient d'un plus fort gain de poids tardif (après 8 semaines d'âge) et n'existe pas chez les poules adultes d'après des données antérieures. Des recherches ultérieures sont suggérées.
\end{abstract}

Mots clés : coqs, poids adulte, gène, crête en pois.

\section{Summary}

Effect of the "pea comb" gene and other genes with visible effects on body weight of adult cocks

In an experimental flock of a medium-heavy brown-egg layer type, from 1961 to 1984 , genotypes at loci controlling pigmentation or morphological traits were compared on pairs of fullor half-sibs. On 42 pairs of $\mathrm{Pp}^{+}$(pea comb) and $\mathrm{p}^{+} \mathrm{p}^{+}$(single comb) cocks, the mean 40 -week body weight of the former genotype exceeds that of the latter by about $250 \mathrm{~g}$, i.e. $7.8 \%$ of the average body weight of the recessive homozygote genotype $(P<0.001)$. This difference comes from larger body weight gain after the age of 8 weeks and is not observed on adult females according to previous data. Further researches are suggested.

Key words : cocks, adult body weight, gene, pea comb.

\section{Introduction}

L'association de gènes à effet visible avec des performances quantitatives chez la poule a été recherchée depuis longtemps ; Mérat (1970) passe en revue les résultats 
antérieurs, ainsi que SMYTH (1969) en ce qui concerne les gènes influant sur la pigmentation du plumage. D'autres résultats ont été obtenus depuis, mais très peu concernent la croissance pondérale des coqs jusqu'au stade adulte : tel est l'objet de la présente note.

\section{Matériel et méthodes}

De 1961 à 1984, un troupeau expérimental mi-lourd du type "pondeuse à œufs teintés » était reproduit annuellement en pedigree, avec choix au hasard des reproducteurs. Un certain nombre de gènes de coloration ou à effet morphologique étaient en ségrégation dans ce troupeau. A l'éclosion de chaque génération, un échantillon d'environ 50 coquelets était gardé et élevé au sol jusqu'à l'âge de 16 semaines, puis en cages collectives ( 7 à 8 coqs par cage) jusqu'à l'âge de 40 semaines. Les coqs étaient pesés à cet âge. L'aliment distribué contenait d'abord $18 \%$ de protéines totales et $2850 \mathrm{kcal} / \mathrm{kg}$ d'énergie métabolisable, puis à partir de 16 semaines d'âge, environ $15 \%$ de protéines totales et $2500 \mathrm{kcal} / \mathrm{kg}$ d'énergie métabolisable. Les génotypes étaient identifiés à l'éclosion, puis revus à l'âge de 8 semaines. Pour leur description, nous nous référons à HUTT (1949) et pour les symboles utilisés à Somes (1984). Les génotypes comparés pour chaque locus figurent au tableau 1. Nous nous sommes restreints aux descendances des familles dont l'un des parents était hétérozygote et l'autre homozygote récessif, ce qui représentait la majorité des données disponibles. Chaque comparaison était faite sur des couples de frères (dans plus de $80 \%$ des cas) ou demi-frères de même père, de sorte que leur génome, hormis le locus étudié et la région chromosomique voisine, était aussi semblable que possible en moyenne. Ceci vaut en particulier pour les locus de coloration ou à effet morphologique envisagés ici, ces derniers ne présentant pas de linkage entre eux (Somes, 1984) et, de plus, les deux frères ou demi-frères d'un couple donné étant le plus souvent du même génotype pour les locus identifiés autres que celui impliqué dans la comparaison. Les locus retenus sont ceux pour lesquels au moins 15 couples étaient présents au total. Pour chacun, la moyenne des différences entre membres d'un couple pour le poids à 40 semaines est comparée à la valeur zéro par un test $\mathrm{t}$ (méthode des couples).

\section{Résultats}

Le tableau 1 résume les comparaisons pour le poids à l'âge de 40 semaines.

Deux résultats apparaissent significatifs : les coqs à pattes et peau blanches $\left(W^{+} \mathbf{w}\right)$ sont un peu plus lourds au stade adulte que ceux à peau jaune (w w); les coqs à crête en pois $\left(\mathrm{Pp}^{+}\right)$ont, au même stade, un poids nettement plus élevé que ceux à crête simple $\left(\mathrm{p}^{+} \mathrm{p}^{+}\right)$. Pour le premier résultat, il paraît raisonnable d'attendre sa confirmation sur des données complémentaires, son niveau de signification $(\mathrm{P}<0,05)$ n'excluant pas qu'il puisse apparaître par hasard sur les 8 comparaisons du tableau. Par contre, il est difficile d'invoquer le hasard de l'échantillonnage, même parmi 8 tests, pour l'effet associé au gène "crête en pois ", très hautement significatif. Sur les 42 couples 
TABleaU 1

Poids à l'áge de 40 semaines de coqs de génotypes différant pour des caractères de pigmentation ou morphologiques

Forty-week body weight of cocks differing by genotype at loci controlling pigmentation or morphological traits

\begin{tabular}{|c|c|c|c|c|c|}
\hline \multicolumn{2}{|c|}{ Génotypes } & \multirow{2}{*}{$\begin{array}{l}\text { Nombre } \\
\text { de couples }\end{array}$} & \multicolumn{2}{|c|}{ Poids moyen (g) } & \multirow{2}{*}{$\begin{array}{c}t \text { et } \\
\text { signification }\end{array}$} \\
\hline (1) & (2) & & (1) & (2) & \\
\hline $\begin{array}{c}\mathrm{C}^{+} \mathrm{c} \\
\text { (plumage coloré) }\end{array}$ & $\begin{array}{l}c c \\
\text { (blanc récessif) }\end{array}$ & 34 & 3361 & 3279 & 1,18 \\
\hline$\stackrel{(E)}{\text { (plumage noir) }}$ & $\begin{array}{l}\text { absence de } E \\
\text { (plumage à noir } \\
\text { restreint) }\end{array}$ & 42 & 3417 & 3375 & 1,05 \\
\hline $\begin{array}{c}\text { B1 b1 } \text { b1 }^{+} \\
\text {(noir dilué } \\
\text { dans le plumage) }\end{array}$ & $\begin{array}{c}\mathrm{b} 1^{+} \mathrm{b} 1^{+} \\
\text {(noir non dilué) }\end{array}$ & 24 & 3223 & 3243 & 0,34 \\
\hline $\begin{array}{c}\mathrm{Bb}^{+} \\
\text {(plumage avec } \\
\text { barrures blanches) }\end{array}$ & $\underset{\text { (plumage non }}{\mathbf{b}^{+} \mathbf{b}^{+}}$ & 15 & 3369 & 3471 & 1,16 \\
\hline $\begin{array}{c}\mathrm{Pp}^{+} \\
\text {(crête en pois) }\end{array}$ & $\begin{array}{l}\mathrm{p}^{+} \mathrm{p}^{+} \\
\text {(crête simple) }\end{array}$ & 42 & 3529 & 3275 & $4,38^{* * *}$ \\
\hline $\begin{array}{l}\mathrm{Rr}^{+} \\
\text {(crête en rose) }\end{array}$ & $\begin{array}{c}\mathbf{r}^{+} \mathbf{r}^{+} \\
\text {(crête simple) }\end{array}$ & 52 & 3535 & 3431 & 1,78 \\
\hline $\begin{array}{c}\mathrm{Dd}^{+} \\
\text {(crête double) }\end{array}$ & $\begin{array}{c}\mathrm{d}^{+} \mathrm{d}^{+} \\
\text {(crête simple) }\end{array}$ & 17 & 3376 & 3472 & 1,24 \\
\hline $\begin{array}{c}\mathrm{W}^{+} \mathbf{w} \\
\text { (peau blanche) }\end{array}$ & $\begin{array}{c}w w \\
\text { (peau jaune) }\end{array}$ & 78 & 3446 & 3344 & $2,21^{*}$ \\
\hline
\end{tabular}

*; ${ }^{* * *}$ : respectivement $\mathrm{P}<0,05 ; \mathrm{P}<0,001$.

Ecart-type : de 338 à $501 \mathrm{~g}$ selon la comparaison (en particulier, l'écart-type pour les coqs $\mathrm{Pp}^{+}$et $\mathrm{p}^{+} \mathrm{p}^{+}$est respectivement 405 et $\mathbf{4 4 2} \mathrm{g}$ ).

obtenus, 32 montrent une différence en faveur du phénotype «crête en pois ». La supériorité pondérale du génotype $\mathrm{Pp}^{+}$, voisine de $250 \mathrm{~g}$, représente près de $8 \%$ du poids moyen du génotype homozygote récessif.

Pour le poids à 8 semaines des mêmes animaux, par contre, il n'existe pas de différence significative entre génotypes : $1009 \mathrm{~g}$ en moyenne pour les coquelets $\mathrm{Pp}^{+}$, $1020 \mathrm{~g}$ pour le génotype $\mathrm{p}^{+} \mathrm{p}^{+}$. D'autre part, sur un troupeau fixé pour le gène de nanisme lié au sexe $\mathrm{dw}, 13$ couples de coqs supplémentaires, l'un $\mathrm{Pp}^{+}$, l'autre $\mathrm{p}^{+} \mathrm{p}^{+}$, sont utilisables entre 1976 et 1983 . Leurs poids moyens à 40 semaines étaient respectivement $2332 \mathrm{~g}$ et $2346 \mathrm{~g}$ (N.S.). Il semblerait, si l'on en juge par cet échantillon très restreint, que l'effet associé au gène $P$ chez des coqs de taille normale n'existe peut-être pas en présence du génotype «nain " $d w d w$ : une analyse de variance en détaillant les facteurs « lignée » (naine ou normale), couple intra-lignée, génotype (crête en pois ou simple $)$, indique une interaction génotype $\times$ lignée significative au seuil $5 \%(F=4,71$ pour 1 d. d.l.). 


\section{Discussion}

L'absence de différence de poids jusqu'à l'âge de 8 semaines associée au gène $P$ concorde avec des résultats antérieurs (Goodman \& Muir, 1965 ; Siegel \& Dudley, 1963 ; HaRTMANN, 1972 ; MoRrison, 1974) alors que d'autres signalent un léger effet dépressif du gène $P$ sur la croissance des premières semaines, effet parfois variable suivant la population considérée (KAN et al., 1959 ; Collins et al., 1961, 1963 ; SмITH, 1961 ; MÉrat \& Bordas, 1979). La différence que nous observons au stade adulte provient donc uniquement d'un écart dans la croissance tardive, précédant ou suivant la maturité sexuelle. Par ailleurs, il paraît peu vraisemblable que le gain de poids tardif plus élevé des coqs à crête en pois reflète un engraissement plus grand, car l'engraissement étant dans l'ensemble plus important chez les femelles, on devrait s'attendre à observer un écart de poids encore plus accentué chez les poules adultes; or le poids moyen des poules adultes $\mathrm{Pp}^{+}$et $\mathrm{p}^{+} \mathrm{p}^{+}$ne diffère pas (Mérat \& Bordas, 1979) et, par ailleurs, leur maturité sexuelle non plus. D'un autre point de vue, le gène "crête en pois » accroît le dimorphisme sexuel pour la taille adulte, au moins en l'absence du gène de nanisme lié au sexe. Une race à crête en pois comme le Cornish a un dimorphisme sexuel accentué, mais elle diffère aussi des autres races par un potentiel de croissance très élevé, de sorte que l'on peut difficilement en tirer une conclusion. D'autre part, parmi les gènes connus, seul le gène de nanisme $d w$ influe sur le dimorphisme sexuel, son effet dépressif étant plus important sur le poids des mâles que sur celui des femelles (cf. revue par Guillaume, 1976) ; il agit donc, précisément, en sens contraire du gène $P$. Il serait souhaitable de comparer ultérieurement certaines activités hormonales et en particulier l'évolution de la production d'androgènes au cours de la vie pour les coqs $\mathrm{Pp}^{+}$et $\mathrm{p}^{+} \mathrm{p}^{+}$. Le fait noté par WiLliams et al. (1977) que les coquelets à crête en pois tendent à être socialement dominés, cependant, ne suggère pas un niveau plus élevé d'androgènes pour le premier génotype. Du point de vue de la reproduction, nous n'avons pas de données suffisantes pour conclure, quoique nos résultats de 1961 à 1984 ne suggèrent pas de différence sensible de fertilité des mâles de ces deux génotypes en insémination artificielle (données non publiées). Ceci n'a pas non plus été mentionné par ailleurs sauf par BuckLand \& Hawes (1968) qui notent un léger effet dépressif du gène «crête en pois » sur ce caractère.

Reçu le 9 février 1987.

Accepté le 13 avril 1987.

\section{Références bibliographiques}

Buckland R.B., Hawes R.O., 1968. Comb type and reproduction in the male fowl. Semen characteristics and testes structure. Poult. Sci., 47, 704-710.

Collins W.H., Hubbard W., Lang B.J., Zervas N.P., 1961. Comb type and body weight of chicken. Poult. Sci., 40, 824-826.

Collins W.H., Lang B.J., Stone H.A., Zervas N.P., Saedeh H.K., 1963. Performance of single and heterozygous pea combed broiler chicken in different brooding environments (abstr.). Poult. Sci., 42, 1260. 
Goodman B.L., Muir F.V., 1965. The influence of comb and feathering phenotypes on body weight and dressing percentage. Poult. Sci., 44, 644-648.

Guillaume J., 1976. The dwarfing gene dw: its effects on anatomy, physiology, nutrition, management, its application in poultry industry. World's Poult. Sci. J., 32, 285-304.

HartmanN W., 1972. Relationship between genes at the pea and single comb locus and economic traits in broiler chicken. Brit. Poult. Sci., 13, 305-309.

Hutt F.B., 1949. Genetics of the fowl. 602 p., McGraw Hill, New York.

Kan J., Manasco B.O., Gyles N.R., Smyth R.M., 1959. The effect of comb type genotypes on six metric traits in a population of meat-type chickens. Poult. Sci., 38, 826-827.

MÉRAT P., 1970. Mendelian genetics and selection for quantitative traits in poultry : results and perspectives. World's Poult. Sci. J., 26, 571-586.

Mérat P., Bordas A., 1979. Effects associated with the pea-comb gene on chick weight and body weight and food efficiency of adult hens. Brit. Poult. Sci., 20, 463-472.

Morrison C.A., 1974. Studies on the occurrence of linkage between the characters breast ridge and pea comb and correlative relationship of breast ridge and body weight at 12 weeks in Cornish White. In : 15th World's Poultry Congress, New-Orleans, August 11-16 1974, 493-495, U.S. Branch, World's Poultry Science Association, Washington, U.S.A.

Siegel P.B., Dudley D.S., 1963. Comb type, behaviour and body weight in chickens. Poult. Sci., 42, 516-522.

Sмiтh J.H., 1961. Relationship between comb type and growth rate in broilers (abstr.). Poult. Sci., 40, 1459.

SMYTH J.R. (Jr.), 1969. Relationship between genes affecting melanin pigmentation and other traits in the fowl. World's Poult. Sci. J., 25, 6-14.

Somes R.G. (Jr.), 1984. International registry of poultry genetic stocks. Storrs Agricultural Experiment Station, Bull. $n^{o} 469,96$ p., University of Connecticut, Storrs, Conn., U.S.A.

Williams C.G., Siegel P.B., Gross W.B., 1977. Social strife in cockerel flocks during the formation of peck rights. Appl. Anim. Ethol., 3, 35-45. 